\title{
Development of CpG ODN Based Vaccine Adjuvant Formulations
}

\author{
Mayda Gursel and Ihsan Gursel
}

\section{Introduction}

The innate immune system responds to the presence of pathogens by sensing "pathogen associated molecular patterns" (PAMPs) expressed by infectious microorganisms [1]. Pathogen-derived nucleic acids represent a critical group of PAMPs that are sensed by a plethora of nucleic acid sensing receptors expressed in immune cells [2]. This recognition initiates a robust innate immune response that enables the host to control the initial spread of infection and subsequently generate sterilizing adaptive immunity. One type of nucleic acid PAMP is the unmethylated CpG motifs present at high frequency in bacterial DNA (but rare in mammalian DNA due to CG suppression and CG methylation) [3]. Unmethylated CpG DNA is recognized by TLR9 expressed by B lymphocytes, dendritic cells (DC), and macrophages. Synthetic oligodeoxynucleotide (ODN) containing unmethylated CpG motifs duplicate the ability of bacterial DNA to stimulate the innate immune system via TLR9 [4].

The immune stimulatory effects of CpG ODNs variegate on the basis of their subcellular distribution, backbone modification, length, and formation of secondary and tertiary structures [5]. Based on their differential activation of immune cells, four major classes of synthetic CpG ODNs have been defined: (a) A or D-type $\mathrm{CpG}$, (b) B or K-type CpG, (c) C-type CpG, and (d) P-type CpG ODNs (Reviewed in ref. 6). In general, K class ODNs are potent $\mathrm{B}$ cell activators and induce TNF- $\alpha$ secretion from plasmacytoid dendritic cells (pDC) but not interferon- $\alpha$. In contrast, D-, C-, and P-class ODNs are capable of stimulating variable amounts of IFN $\alpha$ secretion from pDCs. Of the latter three ODN classes, D ODNs are the most potent IFN $\alpha$ inducers but have the drawback 
of forming multimers, and random concatamers complicating their clinical grade manufacturing process. In fact, to date, only three clinical trials tested the vaccine adjuvant and/or immunotherapeutic activity of D class CpG ODN [7-9]. All three studies harnessed a stabilized version of this ODN class following packaging into virus like particles consisting of the bacteriophage $\mathrm{Q} B$ coat protein.

In this chapter, we describe two alternative methods of preparing $\mathrm{CpG}$ ODN-based vaccine adjuvant formulations that can induce a robust IFN $\alpha$ response from human peripheral blood mononuclear cells. Method 1 details a protocol to stabilize D-type CpG ODN in cationic liposomes. Labile bioagents are protected following liposome encapsulation [10]. This mild approach relies on the dehydration-rehydration technique, does not involve detergents or organic solvents and the encapsulation yield is much higher than most other widely accepted liposome generation methods [11-13].

Method 2 describes a simple strategy of anionic bioactive agent stabilization following complexation with cationic peptides [14-16]. Peptide-mediated multimerization of a K-type ODN devoid of IFN $\alpha$ stimulating activity into stable nuclease-resistant nanostructures (i.e., nanorings) with type I interferon inducing activity is only achieved through the use of a short and non-flexible ODN (K23) and the HIV-derived peptide $\mathrm{Tat}_{(47-57)}$ at a specific ODN-peptide molar ratio (1:16).

\section{Materials}

2.1 CpG ODN

Sequences (Alpha DNA,

Canada: Bases Shown

in Capital Letters Are

Phosphorothioate;

Lower Case Letters

Indicate

Phosphodiester

Backbone)

2.2 Lipids Used

in Liposome

Preparation (Avanti

Polar Lipids,

Alabaster, AL)

2.3 Cationic Peptides

(AnaSpec Inc., USA)
D35 (D-type ODN used in Method 1): GGtgcatcgatgcaggggGG

D35 flip (Control D-ODN with no immunostimulatory activity): GGtgcatgcatgcaggggGG

K23 (K-type ODN used in Method 2): TCGAGCGTTCTC

K23 flip (Control K-ODN with no immunostimulatory activity): TCGAGGCTTCTC

Dimethylaminoethanecarbamol-cholesterol (DC-Chol), dioleoyl phosphatidylethanolamine (DOPE), and polyethylene glycol2000phosphatidylethanolamine (PEG-PE).

\section{LL-37: LLGDFFRKSKEKIGKEFKRIVQRIKDFLRNLVPRTES} HIV-Tat $_{(47-57)}$ : YGRKKRRQRRR 


\subsection{CpG ODN Loaded Liposome Preparation (Method 1)}

2.5 Demonstration of Complexation between CpG ODN and Cationic Peptides Using Agarose Gel Electrophoresis (Method 2)

\subsection{Assessment of Vaccine Adjuvant Formulations for Their IFNo Triggering Activities Using Human Peripheral Blood Mononuclear Cells (hPBMC)}

\subsection{Cytokine Measurement from Culture Supernatants}

1. $50 \mathrm{ml}$ round-bottom flask (Pyrex, vacuum resistant).

2. Rotary evaporator with a water bath attachment (Heidolph, Laborota, Germany, or any brand).

3. Argon cylinder tank (without $\mathrm{O}_{2}$ ).

4. Cup Horn Vibra Cell Sonicator (Sonics and Materials, Danbury, CT, USA, or any brand).

5. Freeze-drier (Heto-Holten, Maxi-Dry Lyo, Denmark, or any brand).

6. LiposoFast extruder equipped with polycarbonate filters (Avestin, Ottawa, Canada).

7. Sterile glass vials $(5 \mathrm{ml})$.

1. Agarose, loading dye, O'GeneRuler 100 bp DNA Ladder (Thermo Scientific, USA), and nucleic acid stain suitable for gel electrophoresis.

2. Agarose gel electrophoresis: for $150 \mathrm{ml}$ of $1.0 \%$ agarose gel, use $1.5 \mathrm{~g}$ of ultrapure agarose (electrophoresis grade) with $150 \mathrm{ml}$ of $1 \times$ TAE. Prepare 11 of $10 \times$ TAE stock solution in ultrapure water with $48.4 \mathrm{~g}$ of Tris base, $3.72 \mathrm{~g}$ disodium EDTA, and adjust to $\mathrm{pH} 8.5$ with glacial acetic acid. Include ethidium bromide $(1 \mathrm{mg} / \mathrm{ml})$ before pouring the gel.

3. Gel documentation system.

1. Ficoll-Paque PLUS density gradient medium (GE Healthcare Biosciences, Sweden).

2. Centrifuge with swing bucket clinical rotor.

3. 96-well tissue culture plates.

4. RPMI-1640 cell culture medium containing $10 \% \mathrm{FBS}, 50 \mu \mathrm{g} /$ $\mathrm{ml}$ penicillin/streptomycin, $10 \mathrm{mM} \mathrm{HEPES}, 0.11 \mathrm{mg} / \mathrm{ml} \mathrm{Na}$ pyruvate, $2 \mathrm{mMl}$-glutamine, $1 \times$ nonessential amino acids (from a $100 \times$ stock solution), and $0.05 \mathrm{mM} 2$-mercaptoethanol.

1. Immulon 2B plates (Thermo Labsystems, USA).

2. Human IFN- $\alpha 2$ ELISA development kit (ALP) from Mabtech, Sweden.

3. ELISA blocking buffer: Phosphate buffered saline (PBS; $10 \mathrm{mM}$ phosphate buffer, $\mathrm{pH} 7.4,150 \mathrm{mM} \mathrm{NaCl}$ ) containing $5 \%$ bovine serum albumin and $0.025 \%$ Tween 20 .

4. ELISA washing buffer: PBS containing $0.05 \%$ Tween 20.

5. Detection antibody diluent: PBS containing $5 \%$ FBS and $0.025 \%$ Tween 20 .

6. SIGMAFAST p-Nitrophenyl phosphate ( $\mathrm{p}$-Npp) substrate tablets.

7. 96-well multi-plate reader equipped with a $405 \mathrm{~nm}$ filter. 


\section{Methods}

\subsection{Preparation of Cationic Liposome Stabilized D-Type CpG ODN (See \\ Scheme 1a, b)}

1. Prepare lipid stocks in chloroform $(10 \mathrm{mg} / \mathrm{ml})$ and store under argon gas at $-20^{\circ} \mathrm{C}$ until use.

2. For the preparation of $20 \mu \mathrm{mol}$ cationic stealth liposomes, pipette lipids from corresponding lipid stocks at a 4:6:0.06 molar ratio (DC-Chol-DOPE-PEG-PE) into a $50 \mathrm{ml}$ round bottom flask.

3. Complete the volume to $2.0 \mathrm{ml}$ by adding chloroform and connect the flask to a rotary evaporator.

4. Set the evaporator rotation speed to maximum (the temperature of the water bath should be set to $37^{\circ} \mathrm{C}$ ).

5. Evaporate the chloroform in the round bottom flask for $20 \mathrm{~min}$.

6. Solvent-free thin lipid film should appear in the inner wall of the round bottom flask at the end of this process.

7. Remove the flask from the rotary evaporator and purge with argon for 30-60 s. Make sure all residual chloroform is removed from the flask and argon purging will remove residual oxygen remained in the flask ( see Note $\mathbf{1}$ ).

8. Seal the round bottom flask with a glass cap and continue the following steps under laminar hood. Transfer 30-40 sterile glass beads ( $300 \mu \mathrm{m}$ average size, from Sigma) into flask.

9. Add $1.0 \mathrm{ml}$ sterile phosphate-buffered saline (PBS) onto beads, and shake the solution in a circular motion until lipid film disappears from the flask wall. This motion helps the lipid film to be removed by the abrasive force of the glass beads and leads to the generation of empty, large multilamellar liposomes.

10. Collect the resulting milky solution from the flask and transfer into a glass vial.

11. In order to generate small unilamellar vesicles (SUVs), sonicate the liposome solution five cycles $(30 \mathrm{~s} /$ cycle) with an amplitude of $70 \%$ and a second set of five cycles with an amplitude of $50 \%$ on ice. Keep the vial on ice for $15 \mathrm{~s}$ in between sonication cycles to prevent excessive heating.

12. For a $20 \mu \mathrm{mol}$ SUV liposome solution (1.0 ml in PBS) add $1 \mathrm{mg}$ CpG ODN solution ( $1 \mathrm{mg} / \mathrm{ml}$ ODN solution) and mix gently by vortexing. Total volume is $2.0 \mathrm{ml}$ at this stage.

13. Remove the vial cap and seal the vial mouth with a Parafilm. Using a syringe needle, punch 6-8 holes on the Parafilm. This will let air out during the lyophilization step.

14. Immediately freeze the liposome/ODN solution in liquid nitrogen for $1 \mathrm{~min}$. 
a

Add phospholipids dissolved in chloroform

Add glass beads and agitate the

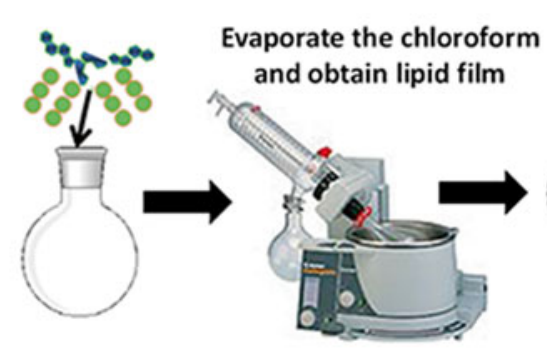

lipid film in PBS to obtain MLVs

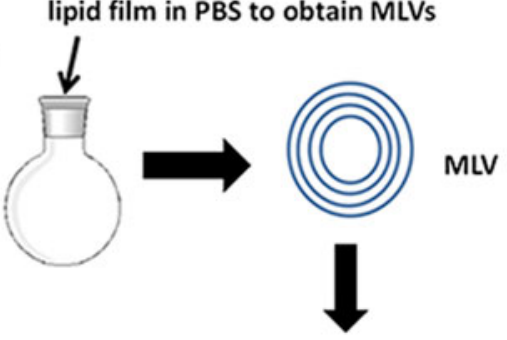

Sonicate to obtain small unilamellar vesicles

(SUV) from multilamellar vesicles (MLV)

Preformed empty SUV ready for nucleic acid loading

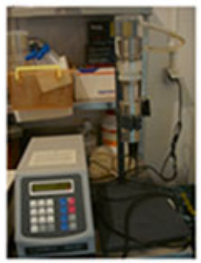

b

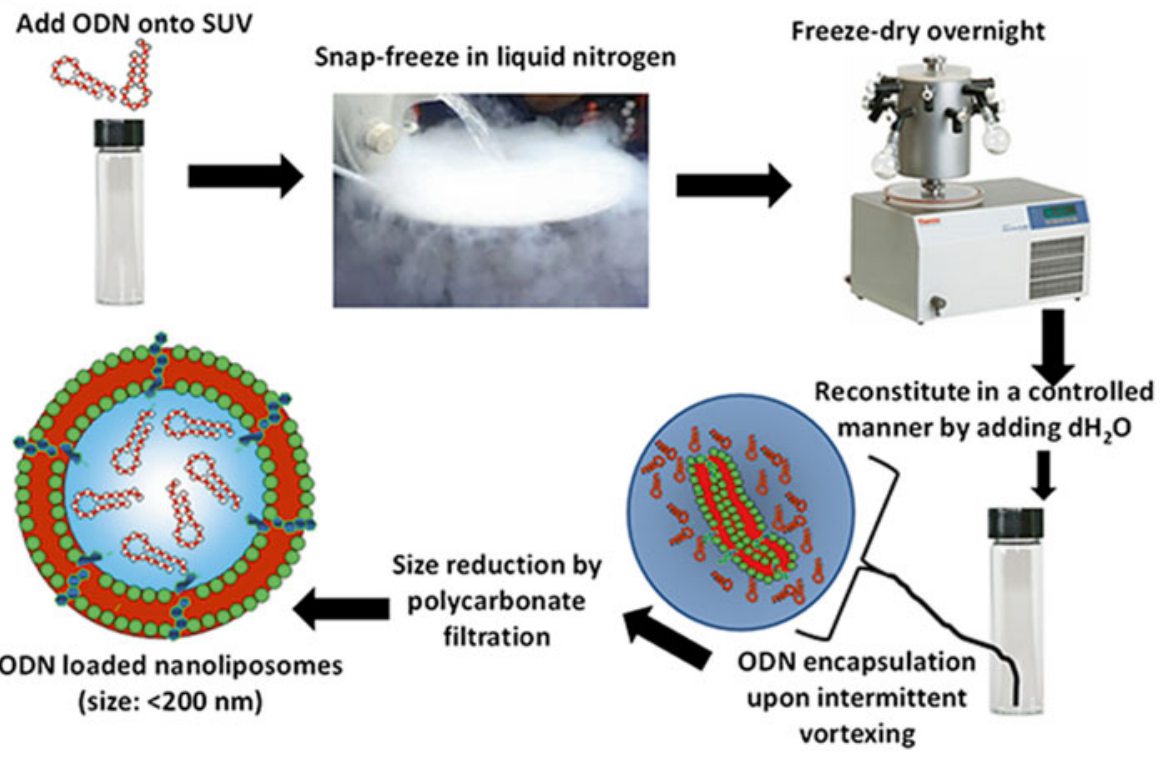

Scheme 1 Preparation of cationic liposome stabilized D-type CpG ODN. (a) Method for the preparation of preformed, unloaded small unilamellar vesicles. (b) Method to generate CpG ODN loaded liposomes 
3.1.1 Determination of ODN Encapsulation Efficiency

\subsection{Preparation and Testing of K-Type CpG ODN/Cationic Peptide Complexes (Method 2)}

3.2.1 Preparation of $\mathrm{CpG}$ ODN/Cationic Peptide Complexes
15. Place the frozen liposome/ODN mixture in a freeze-dryer and lyophilize overnight ( see Note 2).

16. Remove the vial from the lyophilizer. At this stage there should be a white powder in the vial.

17. Add 1:10 volume of $\mathrm{ddH}_{2} \mathrm{O}\left(200 \mu \mathrm{lddH_{2 }} \mathrm{O}\right)$ on to the liposome powder and vortex vigorously for $15 \mathrm{~s}$.

18. Continue vortexing for $15 \mathrm{~s}$ every $5 \mathrm{~min}$ for the total duration of $30 \mathrm{~min}$. This will allow the ODN to dissolve in $\mathrm{ddH}_{2} \mathrm{O}$ and diffuse into the liposome bilayer while liposomes are swelling in the aqueous environment.

19. Add $200 \mu \mathrm{l}$ PBS on to the liposome solution, gently vortex, and set aside for $10 \mathrm{~min}$.

20. Complete the volume to $1.0 \mathrm{ml}$ by adding $600 \mu \mathrm{l}$ PBS. This generates the CpG ODN loaded liposome stock.

21. To reduce the size of the loaded liposomes, assemble the LiposoFast extruder, and gently transfer the liposome solution into the glass syringe provided with the extruder. Filter ten times through the $1.0 \mu \mathrm{m}$ cut-off polycarbonate filter. Replace the filter with the $500 \mathrm{~nm}$ polycarbonate filter and filter 10 more times. Finally, replace $500 \mathrm{~nm}$ filter with the $200 \mathrm{~nm}$ filter and repeat 10 more filtrations.

22. Transfer the extruded nanoliposomes encapsulating the $\mathrm{CpG}$ ODN into a sterile vial.

1. Remove $50 \mu \mathrm{l}$ of the liposome aliquot into a microcentrifuge tube.

2. Centrifuge for $1 \mathrm{~h}$ at $16,100 \times g$ in an Eppendorf centrifuge.

3 . Gently collect the clear supernatant into a clean microcentrifuge tube.

4. Determine the non-encapsulated ODN concentration in the supernatant by recording the OD at $260 \mathrm{~nm}$ using NanoDrop ${ }^{\circledR}$ ND-100 (NanoDrop Technologies, USA).

5. Determine ODN encapsulation efficiency indirectly by subtracting the amount of non-encapsulated ODN from the original input amount and then divide it to the original input ODN amount that was initially mixed with empty SUVs before freeze-drying. Multiply by 100 (see Note 3).

1. Prepare stock solutions of CpG ODNs (K23 and K23 flip) in DNAse-free $\mathrm{ddH}_{2} \mathrm{O}$ (final concentration of $1 \mathrm{mg} / \mathrm{ml}$ ).

2. Prepare stock solutions of cationic peptides in $\mathrm{ddH}_{2} \mathrm{O}$ (final concentration of $5 \mathrm{mg} / \mathrm{ml}$ ).

3. Mix the ODNs and peptides at different molar ratios $(1: 1,1: 2$, $1: 4,1: 8,1: 16$ ) as detailed in Table 1 (see Note 4).

4. Incubate complexes for $30 \mathrm{~min}$ at RT and proceed to confirmation of complexation with agarose gel electrophoresis. 
Table 1

Concentrations of K-type CpG ODN and cationic peptides required to form complexes of various molar ratios

\begin{tabular}{|lllllllll}
\hline Samples & $\begin{array}{l}\text { Molar } \\
\text { ratio }\end{array}$ & $\begin{array}{l}\text { K23 } \\
(\boldsymbol{\mu M})\end{array}$ & $\begin{array}{l}\text { Peptide } \\
(\boldsymbol{\mu M})\end{array}$ & $\begin{array}{l}\text { K23 } \\
(\boldsymbol{\mu g})\end{array}$ & $\begin{array}{l}\text { Peptide } \\
(\boldsymbol{\mu g})\end{array}$ & $\begin{array}{l}\text { K23 }(\boldsymbol{\mu l}) \\
\text { stock 1 } \lambda\end{array}$ & $\begin{array}{l}\text { Peptide }(\boldsymbol{\mu l )}) \\
\text { stock 5 } \boldsymbol{\lambda}\end{array}$ & $\begin{array}{l}\mathbf{H}_{\mathbf{2}} \mathbf{0} \\
(\boldsymbol{\mu l})\end{array}$ \\
\hline K23 & - & 80 & - & 19.2 & - & 19.2 & - & 40.8 \\
\hline K23/LL37 & $1: 1$ & 80 & 80 & 19.2 & 21.54 & 19.2 & 4.2 & 36.6 \\
\hline K23/LL37 & $1: 2$ & 80 & 160 & 19.2 & 43.14 & 19.2 & 8.4 & 32.4 \\
\hline K23/LL37 & $1: 4$ & 80 & 320 & 19.2 & 86.16 & 19.2 & 16.8 & 24 \\
\hline K23/LL37 & $1: 8$ & 80 & 640 & 19.2 & 172.2 & 19.2 & 34.2 & 6.6 \\
\hline K23/Tat & $1: 2$ & 80 & 160 & 19.2 & 15 & 19.2 & 3 & 37.8 \\
\hline K23/Tat & $1: 4$ & 80 & 320 & 19.2 & 30 & 19.2 & 6 & 34.8 \\
\hline K23/Tat & $1: 8$ & 80 & 640 & 19.2 & 60 & 19.2 & 12 & 28.8 \\
\hline K23/Tat & $1: 16$ & 80 & 1280 & 19.2 & 120 & 19.2 & 24 & 16.8 \\
\hline
\end{tabular}

3.2.2 Demonstration of Complexation Using

Agarose Gel

Electrophoresis
3.2.3 Testing of IFNoInducing Activity of Vaccine Adjuvant Formulations Using hPBMC
1. To confirm that $\mathrm{CpG}$ ODN formed complexes with the cationic peptides, mix $20 \mu \mathrm{l}$ of each complex (concentration based on ODN amount) with $4 \mu \mathrm{l}$ of $6 \times$ loading dye and load the wells of a $1 \%$ agarose gel containing $1 \mathrm{mg} / \mathrm{ml}$ ethidium bromide with the samples.

2. Apply uncomplexed CpG ODN (1.6 $\mu \mathrm{g})$ to one well as the negative control.

3. Apply the 100-1000 bp range DNA ladder as a marker $(3 \mu \mathrm{g} /$ well).

4. Carry out agarose gel electrophoresis using $\mathrm{l} \times \mathrm{TAE}$ buffer at $70 \mathrm{~V}$ for $60 \mathrm{~min}$.

5. Visualize the gels under a UV transilluminator (see Note 5).

1. Collect blood samples $(10 \mathrm{ml})$ from healthy donors into anticoagulant containing (sodium citrate, EDTA, or heparin) tubes (Note that blood collection from healthy donors requires ethical approval).

2. Dilute to $20 \mathrm{ml}$ with $1 \times$ PBS.

3. Pipette $10 \mathrm{ml}$ of Ficoll-Paque PLUS density gradient medium into a $50 \mathrm{ml}$ conical tube and carefully layer the diluted blood on top of the gradient medium without disturbing the layers.

4. Centrifuge samples at $400 \times g$ for $30 \mathrm{~min}$ with the break off at room temperature.

5. Using a sterile pipette collect the cloudy PBMC layer that resides at the interphase of the uppermost plasma and the clear density gradient medium and transfer to a new tube. 


\subsubsection{Cytokine ELISA}

6. Wash the cells two times using $50 \mathrm{ml}$ RPMI medium and centrifugation at $400 \times g$ for $10 \mathrm{~min}$.

7. Resuspend the resultant cell pellet in $1 \mathrm{ml}$ of RPMI, count the cells using a hemocytometer and adjust the working cell concentration to $4 \times 10^{6}$ cells $/ \mathrm{ml}$.

8. For testing of the $\mathrm{CpG}$ ODN/cationic peptide complexes, stimulate cells in a 96-well tissue culture plate $(400,000$ cells/well) in a total volume of $200 \mu \mathrm{l}$ using three different doses $(0.3,1$, and $3 \mu \mathrm{M}$ ) of uncomplexed or complexed CpG ODNs and their flip controls for $24 \mathrm{~h}$ at $37^{\circ} \mathrm{C}$ and $5 \% \mathrm{CO}_{2}$ (see Note 6).

9. Collect culture supernatants at the end of this incubation period.

1. Coat a 96-well Immunol II plate using $50 \mu \mathrm{l}$ of anti-human coating antibody in PBS $(5 \mu \mathrm{g} / \mathrm{ml})$.

2. Tap the plates to ensure uniform spreading and incubate at RT for 4 h or at $4{ }^{\circ} \mathrm{C}$ overnight.

3 . Remove the coating solution by inverting the plates, add blocking buffer $(200 \mu \mathrm{l})$ and incubate at RT for $2 \mathrm{~h}$.

4. Decant the blocker, wash plates with ELISA wash buffer five times (immerse plates into a container filled with wash buffer to fill all wells and incubate for 5 min before decanting).

5. Rinse plates with $\mathrm{ddH}_{2} \mathrm{O}$ and dry wells by tapping over an absorbent tissue paper.

6. Distribute $50 \mu \mathrm{l}$ of supernatants and the provided cytokine standard in triplicate $(250 \mathrm{ng} / \mathrm{ml}$ highest concentration; serially diluted twofold in PBS to make up a standard curve of 12 different concentrations) and incubate for $2-3 \mathrm{~h}$ at room temperature or overnight at $4{ }^{\circ} \mathrm{C}$.

7. Wash plates as described above (steps 4 and $\mathbf{5}$ ).

8 . Add $50 \mu$ of $1: 1000$ diluted (dilution in detection antibody diluent) biotinylated-secondary antibody solution into wells and incubate $2-3 \mathrm{~h}$ at room temperature or overnight at $4{ }^{\circ} \mathrm{C}$.

9. Wash plates as described above (steps 4 and $\mathbf{5}$ ).

10. Distribute $50 \mu \mathrm{l}$ of 1:5000 diluted (dilution in detection antibody diluent) streptavidin-alkaline phosphatase solution to each well (see Note 7) and incubate $1 \mathrm{~h}$ at RT.

11. Wash plates as described above (steps 4 and $\mathbf{5}$ ).

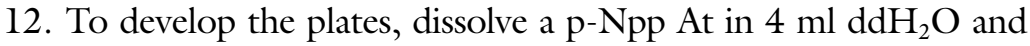
$1 \mathrm{ml} \mathrm{p}$-Npp buffer and transfer $50 \mu$ of this solution to each well.

13. Follow color development at $405 \mathrm{~nm}$ over time using a 96-well multiplate reader until recombinant cytokine standards reach a four-parameter saturation and yield an S-shaped curve. Determine cytokine concentration of each sample using the standard curve (see Note 8). 


\section{Notes}

1. This step is critical. Argon purging eliminates both residual chloroform and also replaces the oxygen present in the flask. $\mathrm{O}_{2}$ gas facilitates lipid peroxidation, so it is vital to remove all oxygen in the flask via argon purging.

2. At this stage, there is no encapsulation of ODN within the liposome. The encapsulation will be achieved during the dehydration-rehydration step).

3. Expected encapsulation efficiency for the D ODN should be at least $80 \%$ or higher. The activity of as such prepared liposomes can be tested as described in Subheading 3.2.2 prior to mixing with an antigen of choice for vaccination experiments.

4. Preparation of complexes in salt containing buffers compromises complexation efficiency. Final volume of the solution in which complexes are formed should not exceed $60 \mu$ l. Table 1 details the optimal volumes and concentrations of reagents to be used for the most efficient complexation.

5. Expected results are demonstrated in Fig. 1.

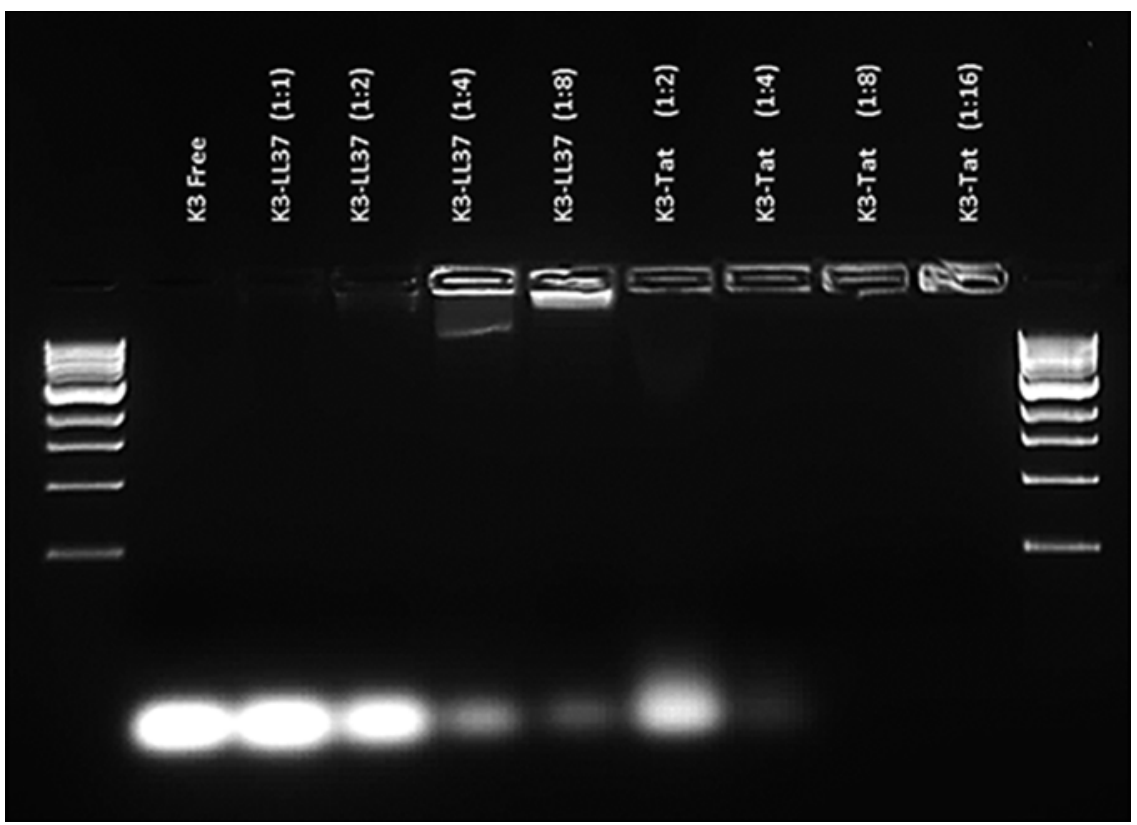

Fig. 1 A constant amount of K-ODN $(80 \mu \mathrm{M})$ was incubated with increasing amounts of cationic peptides for $30 \mathrm{~min}$ at room temperature in a final volume of $60 \mu \mathrm{lddH_{2 }} 0$. CpG ODN or its complexes (1.6 $\left.\mu \mathrm{g} / \mathrm{well}\right)$ were subjected to agarose gel electrophoresis. Uncomplexed CpG ODN demonstrates a bright signal at the bottom of the gel whereas this signal disappears following successful complexation. DNA ladder with 100-1000 bp range was used as a marker ( $3 \mu \mathrm{g} /$ well) 
6. For example, for the $3 \mu \mathrm{M}$ final ODN concentration, mix $48.6 \mu \mathrm{l}$ of formed complex with $275.4 \mu \mathrm{l}$ RPMI medium and add $50 \mu \mathrm{l}$ of this onto $150 \mu$ l cells.

7. The streptavidin-alkaline phosphatase solution must be prepared at least $2 \mathrm{~h}$ prior to its use to ensure uniform color development.

8. We found that $\mathrm{K} 23$ :Tat $(1: 16 ; 1 \mu \mathrm{M})$ triggered an interferonalpha response that was equivalent to levels obtained with $3 \mu \mathrm{M}$ D ODN stimulation. LL-37-incorporating aggregates elicited a substantially lower response.

\section{Acknowledgements}

This work was supported by TÜBİTAK grants $113 S 207$ to I.G, $113 S 305$ and $111 S 151$ to M.G.

\section{References}

1. Medzhitov R (2007) Recognition of microorganisms and activation of the immune response. Nature 449:819-826

2. Kumar H, Kawai T, Akira S (2011) Pathogen recognition by the innate immune system. Int Rev Immunol 30:16-34

3. Krieg AM, Yi AK, Matson S, Waldschmidt TJ, Bishop GA, Teasdale R, Koretzky GA, Klinman DM (1995) CpG motifs in bacterial DNA trigger direct B-cell activation. Nature 374:546-549

4. Klinman DM, Currie D, Gursel I, Verthelyi D (2004) Use of CpG oligodeoxynucleotides as immune adjuvants. Immunol Rev 199:201-216

5. Hanagata N (2012) Structure-dependent immunostimulatory effect of CpG oligodeoxynucleotides and their delivery system. Int J Nanomedicine 7:2181-2195

6. Bode C, Zhao G, Steinhagen F, Kinjo T, Klinman DM (2011) CpG DNA as a vaccine adjuvant. Expert Rev Vaccines 10:499-511

7. Senti G, Johansen P, Haug S, Bull C, Gottschaller C, Muller P, Pfister T, Maurer P, Bachmann MF, Graf N, Kündig TM (2009) Use of A-type CpG oligodeoxynucleotides as an adjuvant in allergen-specific immunotherapy in humans: a phase I/IIa clinical trial. Clin Exp Allergy 39:562-570

8. Klimek L, Willers J, Hammann-Haenni A, Pfaar O, Stocker H, Mueller P, Renner WA, Bachmann MF (2011) Assessment of clinical efficacy of CYT003-QbG10 in patients with allergic rhinoconjunctivitis: a phase IIb study. Clin Exp Allergy 41:1305-1312

9. Beeh KM, Kanniess F, Wagner F, Schilder C, Naudts I, Hammann-Haenni A, Willers J, Stocker
H, Mueller P, Bachmann MF, Renner WA (2013) The novel TLR-9 agonist QbG10 shows clinical efficacy in persistent allergic asthma. J Allergy Clin Immunol 131:866-874

10. Gregoriadis G (2007) Liposome technology. Informa Healthcare, New York

11. Gregoriadis G, Gursel I, Gursel M, McCormack B (1996) Liposomes as immunological adjuvants and vaccine carriers. J Control Release 41:49-56

12. Gursel I, Gursel M, Ishii KJ, Klinman DM (2001) Sterically stabilized cationic liposomes improve the uptake and immunostimulatory activity of $\mathrm{CpG}$ oligonucleotides. J Immunol 167:3324-3328

13. Erikçi E, Gursel M, Gursel I (2011) Differential immune activation following encapsulation of immunostimulatory $\mathrm{CpG}$ oligodeoxynucleotide in nanoliposomes. Biomaterials 32:1715-1723

14. Gungor B, Yagci FC, Tincer G, Bayyurt B, Alpdundar E, Yildiz S, Ozcan M, Gursel I, Gursel M (2014) CpG ODN nanorings induce IFN $\alpha$ from plasmacytoid dendritic cells and demonstrate potent vaccine adjuvant activity. Sci Transl Med 6:235ra61

15. Gungor B, Yagci FC, Gursel I, Gursel M (2014) Forging a potent vaccine adjuvant: CpG ODN/ cationic peptide nanorings. Oncoimmunology 3, e950166

16. Yildiz S, Alpdundar E, Gungor B, Kahraman T, Bayyurt B, Gursel I, Gursel M (2015) Enhanced immunostimulatory activity of cyclic dinucleotides on mouse cells when complexed with a cell-penetrating peptide or combined with CpG. Eur J Immunol 45:1170-1179 